\title{
On the Impact of Correlated Shadowing on the Performance of User-in-the-Loop for Mobility
}

\author{
Tamer Beitelmal, Rainer Schoenen, Halim Yanikomeroglu \\ Department of Systems and Computer Engineering, Carleton University, Canada \\ \{tamer, rs, halim\}@sce.carleton.ca
}

\begin{abstract}
The cellular network users are demanding more traffic and expecting a ubiquitous high data rate. Several techniques are investigated to cope with this massive demand. All the current solutions are focusing on improving this issue from the supply side. However it is expected that a significant improvement on the system performance can be gained if the end users become a part of the system and not just consumers. The user-in-the-loop (UIL) spatial approach provides a solution to fulfil the increasing traffic demand by convincing users to move to locations with higher SINR values. The previous results of the UIL are promising and a higher cell spectral efficiency is achieved only by the involvement of the users and without any investment on the supply side. UIL is a user-centric approach that offers an incentive to influence users' behavior to participate in the system improvement process. In this paper, the previous ideal environment of UIL is extended by integrating the important effect of correlated shadowing to the analysis of UIL performance. The obtained results in this paper confirm the previous results and promise significant performance improvements. Results show that the achieved higher spectral efficiency and the required moving distance depend on the correlation parameters.
\end{abstract}

Index Terms-IMT-Advanced; correlated shadowing; user-inthe-loop (UIL); user behavior

\section{INTRODUCTION}

The cellular network data traffic is increasing exponentially and the paradigm of the cellular network is shifting from principally voice-traffic to more data-traffic [1]. The radio section of the International Telecommunication Union (ITU-R) specified the standard for the new generation of the cellular network that is IMT-Advanced [2]. Several techniques were introduced to achieve the high requirements of the new standard and to support the expected exponential growth of data traffic. The current practice is to improve the performance of the cellular network from the supply side (i.e. by implementing new techniques or installing new base stations), however, the demand side stayed unchanged and users are expecting a ubiquitous very high data rate, which is not always viable. A new user-centric paradigm should be considered to motivate users to be an element of the system and not just consumers.

Some reasons of this consideration are as follows:

- This increase of the required data traffic leads to excess of the demand versus supply. The existing techniques on the supply side are reaching their theoretical limit and further improvement is not expected soon.

- The base station (BS) has limited resources shared among several users. It is important to raise users' awareness towards sharing the network services and resources. We cannot rely only on users' good behavior to share the resources appropriately [3].

- Users close to the BS receive much stronger signal power than users located far from the BS. In addition to this distance-dependent power attenuation, there is the shadowing effect that impacts the signal before reaching the receiver and hence users at equal distances from the BS may not receive exactly the same power [4]. If users with weak received power are willing to move to better locations, they will obtain higher data rates and the overall system capacity is increased.

Consequently, an approach to control the users' behavior is needed to cope with the high traffic demand.

User terminals (UTs) at low power locations consume up to 10 times more resources from the BS to get the same data rate as UTs at locations with stronger received power. Therefore, it is clear that higher overall capacity can be achieved if some users cooperate by moving to better locations. Convincing users to participate as a part of the system was proposed as a new approach in [5], [6] and was named User-In-TheLoop (UIL) [7]. The effect of user movement on the cell spectral efficiency was discussed in [8]. By moving a walkable distance, the cell saves some resources that can be used to serve other users or to provide higher data rates for current users. The results in [8] are promising and more than $100 \%$ increase in the spectral efficiency is achieved without any modification to the current system. This improvement was gained merely by the participation of the users as a part of the system. The previous results were based on the pathloss calculation only, which is an ideal scenario.

The motivation of this paper is to apply the UIL approach in a more realistic environment by integrating the effect of the shadowing, which is important in such a user mobility approach as it can affect the moving distance. For this purpose, both uncorrelated and correlated shadowing are considered. The obtained simulation results for the cell spectral efficiency are similar to the previous results. This similarity is an indication that the pdf of the spectral efficiency is converging in both cases (with and without shadowing). The results also show that the average moving distance depends on the shadowing parameters and this knowledge is necessary as it impacts the user attitude towards moving.

The paper is organized as follows: Section II is a general background of the UIL concept. In Section III, the evaluation scenarios are explained. The simulation results are discussed in Section IV while Section V is the conclusion. 


\section{USER IN THE LOOP}

The goal of the UIL approach is to increase the cell spectral efficiency $(\gamma)$ measured in $\mathrm{b} / \mathrm{s} / \mathrm{Hz}$. The cell spectral efficiency is the averaged rate achieved over the cell coverage area and divided by the bandwidth of the system [9]. The spectral efficiency for each UT depends on its received signal to interference-plus-noise ratio (SINR), the higher the SINR the higher the spectral efficiency [8]. UTs with low SINR have low spectral efficiency and acquire more resource blocks (RBs) to obtain high data rates. To increase the cell spectral efficiency, more users must receive higher SINR which might not be possible because of their bad locations and the limitation on the maximum transmitted power. The relationship between the SINR values and the corresponding spectral efficiency $(\gamma)$ is summarized in Table I and based on the adaptive modulation and coding scheme (AMC). The highest $\gamma$ of $5 \mathrm{~b} / \mathrm{s} / \mathrm{Hz}$ is achieved when using the 64-QAM with a code rate of $5 / 6$ that corresponds to the strongest SINR. On the other extreme, a $\gamma$ as low as $2 / 3 \mathrm{~b} / \mathrm{s} / \mathrm{Hz}$ is obtained from QPSK with $1 / 3$ coding rate.

TABLE I

PHY MODES AND SINR INTERVALS.

\begin{tabular}{|c|c|c|c|c|c|c|c|c|}
\hline Index $i$ & 1 & $\overline{2}$ & $\overline{3}$ & 4 & 5 & 6 & 7 & $\overline{8}$ \\
\hline $\begin{array}{l}\text { SINR }[\mathrm{dB}] \\
\end{array}$ & 0.9 & 2.1 & 3.8 & 7.7 & 9.8 & 12.6 & 15.0 & 18.2 \\
\hline Mod. & \multicolumn{3}{|c|}{ QPSK } & \multicolumn{3}{|c|}{ 16-QAM } & \multicolumn{2}{|c|}{ 64-QAM } \\
\hline Cod. rate & $1 / 3$ & $1 / 2$ & $2 / 3$ & $1 / 2$ & $2 / 3$ & $5 / 6$ & $2 / 3$ & $5 / 6$ \\
\hline$\gamma[\mathrm{b} / \mathrm{s} / \mathrm{Hz}]$ & $2 / 3$ & 1 & $4 / 3$ & 2 & $8 / 3$ & $10 / 3$ & 4 & 5 \\
\hline
\end{tabular}

Instead of increasing the SINR for UTs at current locations, a solution is offered by UIL approach [5], [8] to convince users to move to higher SINR locations. The general idea of including users in the loop is shown in Figure 1. The controller provides information to the user who in return should decide to change his location or not.

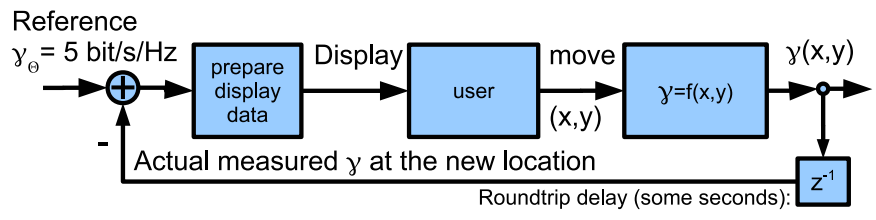

Fig. 1. The user becomes a part of the system (in the loop) [5].

This cooperation from users will provide advantages for the operators because fewer RBs are assigned to users in high SINR locations, consequently they can save resources to better serve current users or accommodate new users. For the benefit of users, the user who is willing to move will get an incentive (a cheaper price or a higher data rate). This movement to a better location is well observed in $\mathrm{Wi}-\mathrm{Fi}$ (IEEE802.11) network where users change their locations to get higher data rates. To facilitate the moving decision for the user, the authors in [5] and [8] suggested that the UT device should have an indicator for the better spectral efficiency locations plus an option to display a map to guide the user to reach the destination. The moving distance in meters, $d$ can be indicated on the device as well. It is understood that not all the users are willing to move, they are free to decide whether or not to change their locations. The probability of
TABLE II

TECHNOLOGY PARAMETERS ACCORDING TO LTE-A.

\begin{tabular}{|c|c|}
\hline Bandwidth & FDD: DL: $20 \mathrm{MHz}$, UL: $20 \mathrm{MHz}$ \\
\hline Traffic & full load; best effort \\
\hline Antenna gain (boresight) & $17 \mathrm{dBi}$ \\
\hline Antenna aperture horizontal $\phi_{3 d B}$ & $70^{\circ}$ \\
\hline Antenna aperture vertical $\phi_{3 d B}$ & $15^{\circ}$ \\
\hline Thermal noise & $-174 \mathrm{dBm} / \mathrm{Hz}$ \\
\hline UT noise figure & $5 \mathrm{~dB}$ \\
\hline
\end{tabular}

TABLE III

UMa SCENARIO SPECIFICATIONS.

\begin{tabular}{|c|c|}
\hline$d_{B S-B S}$ & $500 \mathrm{~m}$ \\
\hline$h_{B S}$ & $25 \mathrm{~m}$ \\
\hline Ant. tilt $\phi_{t}$ & $-12^{\circ}$ \\
\hline$f_{C}$ & $2.0 \mathrm{GHz}$ \\
\hline$P_{T x}$ & $49 \mathrm{dBm}$ \\
\hline$s t d-L O S$ & $4 \mathrm{~dB}$ \\
\hline$s t d-N L O S$ & $6 \mathrm{~dB}$ \\
\hline
\end{tabular}

user movement $\left(p_{M}\right)$ is the probability that a user will move from the current location $\overrightarrow{p_{1}}=\left(x_{1}, y_{1}\right)$ with $\gamma_{1}$ to the new location $\overrightarrow{p_{2}}=\left(x_{2}, y_{2}\right)$ with $\gamma_{2}$ where $\gamma_{2} \geq \gamma_{\Theta} \geq \gamma_{1}$. This $\gamma_{\Theta}$ is chosen to detect the cell spectral efficiency to be achieved after the user movement.

In this paper, moderate values are chosen for the $p_{M}$ and the $\gamma_{\Theta}$ as 0.5 and $2.5 \mathrm{~b} / \mathrm{s} / \mathrm{Hz}$ respectively. $p_{M}=0.5$ means half of the users are willing to walk to better locations. The user moving distance depends on the chosen $p_{M}$ and $\gamma_{\Theta}$ as well as the parameters of the evaluation scenarios.

\section{EVALUATION SCENARIOS}

The evaluation scenarios are presented in this section to analyze the performance of the UIL approach. The Urban Macro-cell (UMa) channel model scenario with its parameters is presented in Subsection A, and the chosen shadowing model to be integrated with the previous UIL approach is explained in Subsection B.

\section{A. IMT-Advanced, UMa Scenario}

In the Urban Macro-cell scenario, the UTs are located outdoor and the BSs are on the roof of the buildings. The line of sight (LOS) signal is not the common path in this environment because of buildings. The general parameters for the channel models of IMT-Advanced are listed in table II. The pathloss $(P L)$ calculation is based on the two pathloss models: LOS or Non-LOS (NLOS) and according to the probability of each of them [4]. The cellular network layout is one cluster with 7 cells with a frequency reuse of 1 . The parameters of this scenario are given in Table III and we consider the case of single-input single-output (SISO) antenna pattern. In addition to the pathloss, the shadowing effect $\left(X_{i}\right)$ is taken as a random value added to the received power $\left(P_{r}\right)$ at the UT. The received power can be calculated in a simple way, and without loss of generality, as

$$
P_{r}[\mathrm{~dB}]=P_{t}+G_{t}+G_{r}-P L+X_{i},
$$

where $P_{t}$ is the transmitted power in $\mathrm{dB}, G_{t}$ is the transmitter antenna gain and $G_{r}$ is the receiver antenna gain. 


\section{B. The Shadowing Model}

The shadowing effect is the result of obstructing the transmitted signal by buildings, natural obstacles, vehicles, etc. The shadowing effect plays an important role in determining the received power (at a UT) from a specific BS. To calculate this effect, a random value is usually added to the received power as shown in (1).

The shadowing effect can be obtained from either independent (uncorrelated) shadowing or correlated shadowing models. In the first model, each point is assumed to have an independent shadowing value $\left(Z_{n} \sim N(0, \sigma)\right)$ represents a Gaussian random variable with a zero mean and a $\sigma \mathrm{dB}$ standard deviation [4]. In this model the shadowing values are taken as independent at each point: that is two neighboring points may have completely different shadowing values despite their adjacency. This independence in shadowing values is an ideal assumption and it will affect the accuracy of the simulation results especially when the number of transmitters/receivers increases [10]. A more realistic model is the correlated shadowing that implies: if a UT is at a deep shadowing, its neighbors probably have deep shadowing as well. The correlation in shadowing can significantly affect the mobility behavior and the received power and hence impacts the overall system performance [10]. UIL approach is based on the user movement, therefore using the correct shadowing model is vital in calculating the moving distance and the resulting spectral efficiency. In such a user mobility approach, it is essential to correctly calculate the distance to move and give an accurate movement suggestion to users and this can be done by choosing a correlated shadowing model instead of the uncorrelated one. Nevertheless, the calculations of the independent shadowing are done to highlight the effect correlated shadowing on the results.

Correlation in shadowing is widely studied in the literature and there are several proposed models. Some models are correlated in distance only while others are considering only the angle between the two points and some took into account both the distance and the angle between any two points to calculate the shadowing value at a specific point considering the correlation among neighbors [11]-[14]. For a small number of receiving/transmitting points, the difference between the uncorrelated shadowing and the correlated shadowing may be neglected, but as the number of terminals increases, the effect of correlation in shadowing is increasing thus it should be considered. For more insight into the difference between the two models, Figures 2 and 3 show a sample of each one. Both of the figures show the value of the shadowing in a 2$\mathrm{D}$ field representing the coverage area for the given scenario. In Figure 2 each point has an independent shadowing value from its neighbors, while in Figure 3 the adjacent points have correlated shadowing values.

In this paper, the correlated shadowing model studied in [10], [15] is used. This model is a two dimensional model, it depends on both the angle $(\phi)$ and the distance ratio $(R$, in $\mathrm{dB})$ between any two points $\overrightarrow{r_{1}}$ and $\overrightarrow{r_{2}}$. The calculation of these two parameters is shown in (2) and (3). This specific shadowing model was chosen because it has two tunable parameters and

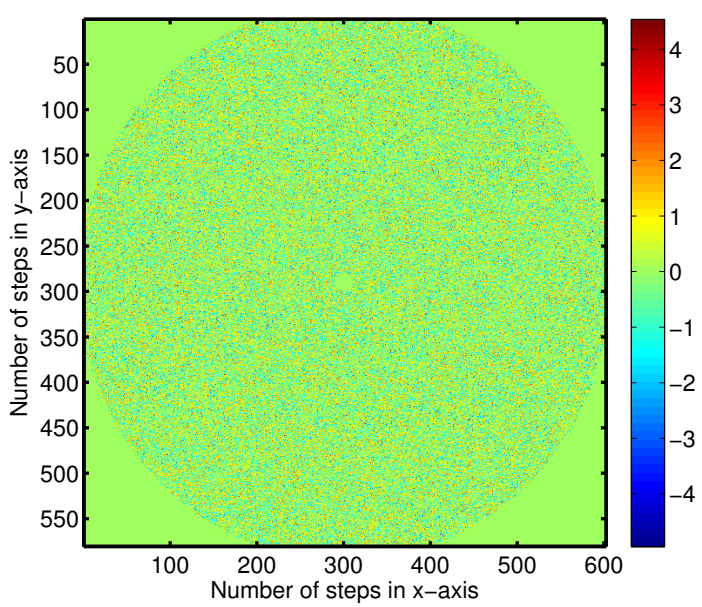

Fig. 2. Example of two-dimensional uncorrelated shadowing field.

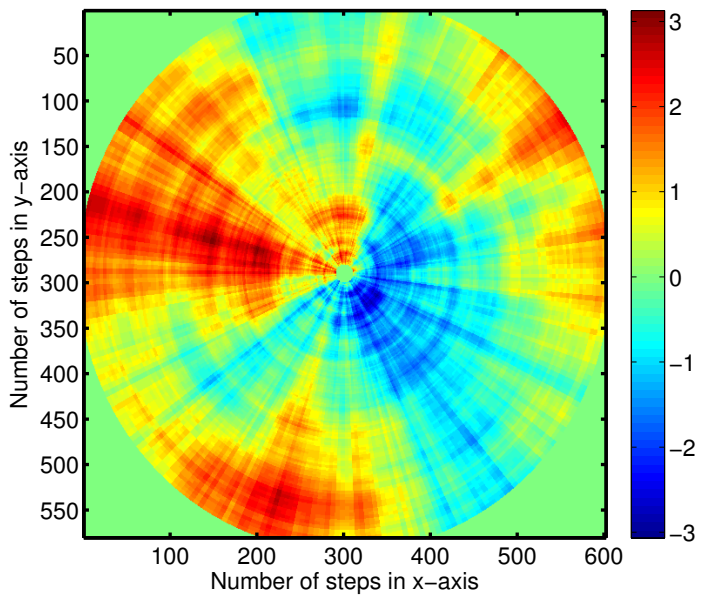

Fig. 3. Example of two-dimensional correlated shadowing field.

it is separable. A separable model is necessary to reduce the simulation complexity especially in this paper to calculate the spectral efficiency all over the coverage area (too many points are considered). Separability means that correlation can be imposed in one dimension first, and then the correlation in the other dimension can be calculated according to (4) [10] as

$$
\begin{gathered}
\phi=\left|\angle \overrightarrow{r_{1}}-\angle \overrightarrow{r_{2}}\right| \in\left[0^{\circ}, 180^{\circ}\right], \\
R[\mathrm{~dB}]=\left|10 \log \left(r_{1} / r_{2}\right)\right|, R_{0}>0, \\
h(\phi, R)=h_{\phi}(\phi) h_{R}(R) .
\end{gathered}
$$

Here is the abstract description of this model: If two UTs are located at the same point then they are $100 \%$ correlated. This correlation ratio decreases as the distance ratio and/or the angle between the UTs increases [10]. After certain values, the two UTs are considered to be totally uncorrelated. These values are defined as $\left(\phi_{0}\right)$ : the maximum angle after which any two UTs are uncorrelated in angle, and $\left(R_{0}\right)$ : the maximum distance ratio after which any two UTs are uncorrelated in distance. This model starts with a set of independent random variables 
to obtain an independent shadowing field (similar to that in Figure 2). After that, it is necessary to obtain the correlation coefficient matrix between any two points $r_{1}$ and $r_{2}$ according to (5). To obtain the correlated shadowing field, the correlation coefficient matrix is applied to the independent shadowing field. Applying this model guarantees that each shadowing value will be correlated with all the neighboring points. After obtaining this correlated shadowing field, the shadowing value at any point can be obtained directly by mapping the $x$ and $y$ values of that point and getting the corresponding shadowing value from the previously obtained correlated shadowing field.

$$
h\left(r_{1}, r_{2}\right)=\max \left\{1-\phi / \phi_{0}, 0\right\} \cdot \max \left\{1-R / R_{0}, 0\right\} .
$$

\section{Simulation Results}

According to section III, the model analysis is done by calculating the cell spectral efficiency before and after applying the UIL mobility and the calculation methodology is as described in [8]. First, it is informative to visualize the effect of correlated shadowing on the SINR map. Figure 4(a) shows the LOS SINR distribution in the ideal case without including the shadowing. In this case, the SINR values depend on the distance and antenna patterns only. The distance-dependent SINR map is changed when the correlated shadowing is included as shown on Figure 4(b). The Importance of correlated shadowing on the distribution of SINR is observed in Figure 4 and consequently its impact on the required moving distance.

The simulation was run for a sufficiently enough number of trials and then the average is calculated. The spectral efficiency values obtained by running our simulation are very similar to those obtained in the original results of the UIL [8]. This similarity in the results is an indication for the convergence of spectral efficiency pdf. Although the SINR distribution is different in the two cases, the average spectral efficiency is the same.

In order to comprehend the effect of shadowing on the moving distance, the results for the independent shadowing model are shown in row 3 in Table IV. This result shows a smaller distance to move compared with the original UIL approach without the shadowing effect (row 2). This distance can be as small as the grid resolution used for the simulation model. In fact this smaller distance is expected with independent shadowing because the user will find at least one adjacent location with $\gamma \geq \gamma_{\Theta}$. To analyze the model in a more realistic scenario, the correlated shadowing model in [10] is applied. In this case the moving distance depends on the values of $\phi_{0}$ and $R_{0}$, which represents the correlation parameters. Different $\phi_{0}$ and $R_{0}$ values were selected to evaluate their impact on the spectral efficiency and the user movement distance. Some results are tabulated in Table IV and they are interpreted as: the smaller the correlation parameters the smaller the distance to move.

Figures 5, 6 and 7 illustrate the relations between the correlation parameters and the corresponding moving distance. As extracted from these figures, the average moving distance is as low as $3.34 \mathrm{~m}$ for small correlation parameters $\left(R_{0}=1\right.$ $\mathrm{dB}$ and $\phi_{0}=3^{\circ}$ ) that is $\sim 30 \%$ shorter than the average moving distance in the original UIL. On the other hand,
TABLE IV

Cell-Average Spectral EFFiciency Results For the UMa SCEnARIO Evaluation $[\mathrm{b} / \mathrm{s} / \mathrm{Hz} /$ Sector $]$ WITH $p_{M}=0.5$ AND $\gamma_{\Theta}=2.5 \mathrm{~b} / \mathrm{s} / \mathrm{Hz}$.

\begin{tabular}{|c|c|c|c|}
\hline Scenario & $\begin{array}{c}\gamma[\mathrm{b} / \mathrm{s} / \mathrm{Hz}] \\
\text { without UIL }\end{array}$ & $\begin{array}{c}\gamma[\mathrm{b} / \mathrm{s} / \mathrm{Hz}] \\
\text { with UIL }\end{array}$ & $\begin{array}{c}\text { Average moving } \\
\text { distance }[\mathrm{m}]\end{array}$ \\
\hline No shadowing effect & 1.254 & 1.974 & 4.7 \\
\hline Uncorrelated shadowing model & 1.225 & 2.028 & 2.69 \\
\hline $\begin{array}{c}\text { Correlated shadowing } \\
R_{0}=1 \mathrm{~dB}, \phi_{0}=3^{\circ}\end{array}$ & 1.227 & 2.041 & 3.34 \\
\hline $\begin{array}{c}\text { Correlated shadowing } \\
R_{0}=3 \mathrm{~dB}, \phi_{0}=30^{\circ}\end{array}$ & 1.234 & 2.016 & 4.69 \\
\hline $\begin{array}{c}\text { Correlated shadowing } \\
R_{0}=6 \mathrm{~dB}, \phi_{0}=60^{\circ}\end{array}$ & 1.220 & 2.005 & 6.06 \\
\hline
\end{tabular}

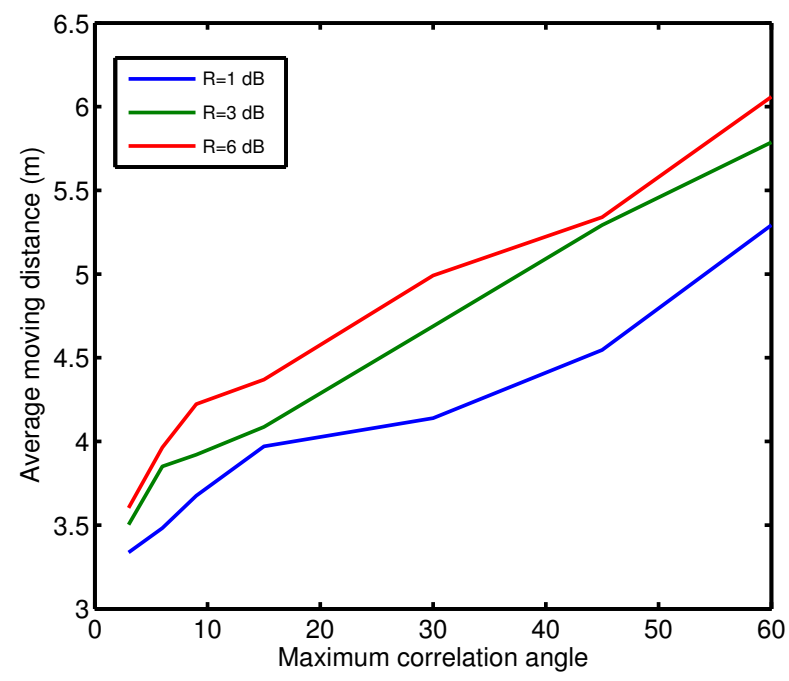

Fig. 5. Moving distance with different correlation angles $\phi_{0}$.

large correlation parameters result in a longer average moving distance (6.06 m compared to $4.7 \mathrm{~m}$ ). However calculated cell spectral efficiency is very similar for the different shadowing cases. This indicates that the probability density function (pdf) of the cell spectral efficiency doesn't change with different correlation parameters.

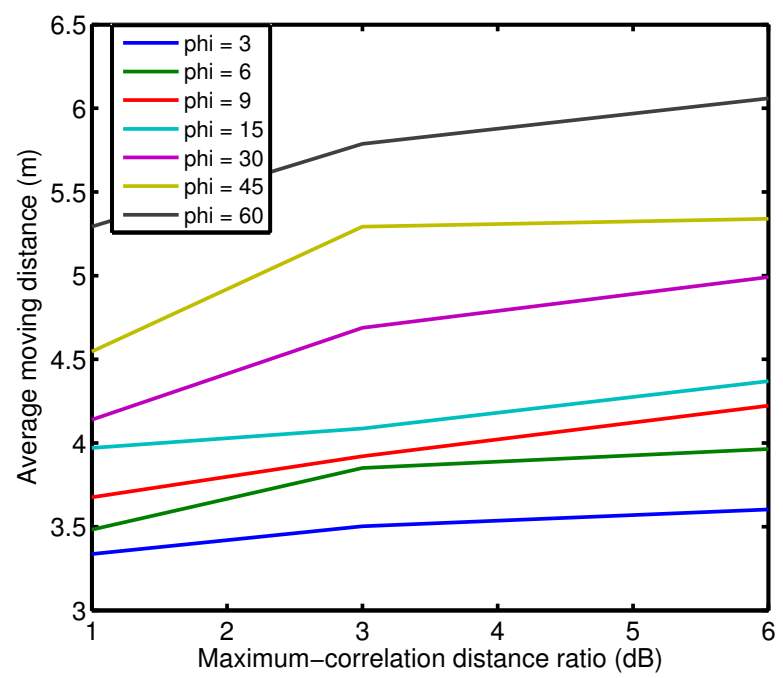

Fig. 6. Moving distance with different correlation distance ratios $R_{0}$. 


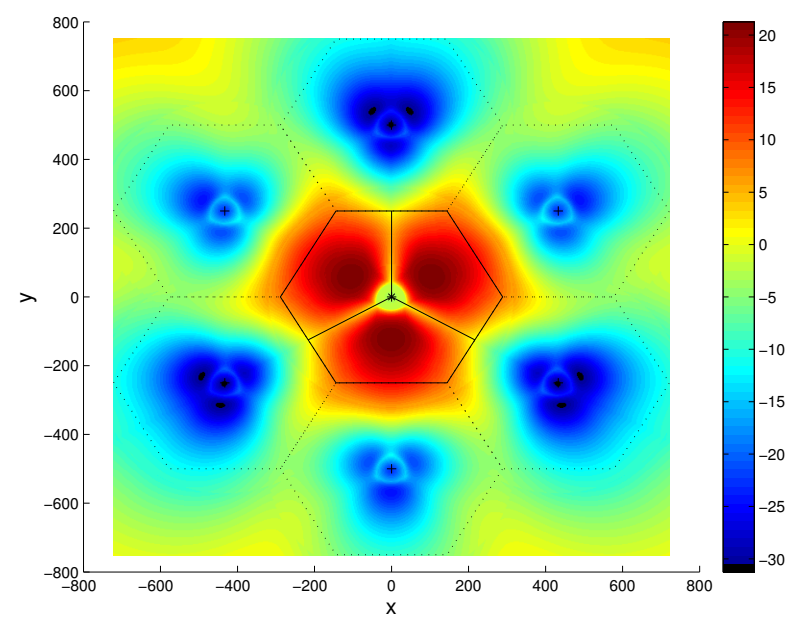

(a) LOS SINR without correlated shadowing.

Fig. 4. Examples of the effect of shadowing on the strength of the SINR.

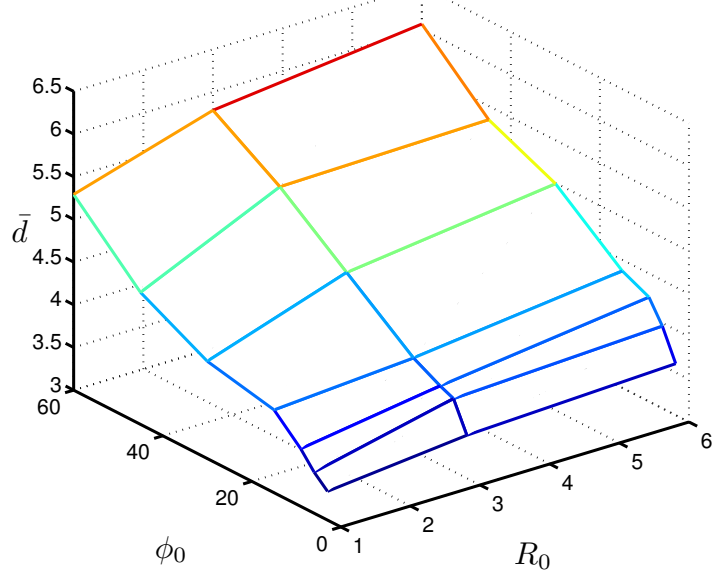

Fig. 7. A 3-D presentation of the relation between the maximum correlation (angle $\phi_{0}$ and distance ratio $R_{0}$ ) and the average moving distance $\bar{d}$.

\section{CONClusion}

The massive growth of data traffic in cellular network requires a paradigm shift to be more user-centric. The UIL approach is a user-centric approach based on convincing users to be part of the system by providing them some incentive. Users can cooperate by moving to higher SINR locations. This user cooperation can lead to a significant increase in the cell spectral efficiency with no change to the current cellular system as shown in the UIL literature. A further investigation was done in this paper by including the shadowing effect to analyze the performance of the UIL approach in a more realistic environment for high precision. The obtained results confirmed the previous promising increase on the spectral efficiency, however, the average moving distance was affected. The results show the relation between the correlation parameters and the average moving distance.

In a parallel work, a survey was conducted to understand the user behavior in relation to different class of incentives [16]. For more accurate results, the $p_{M}$ values suggested in [16] can be used instead of 0.5 used in this paper.

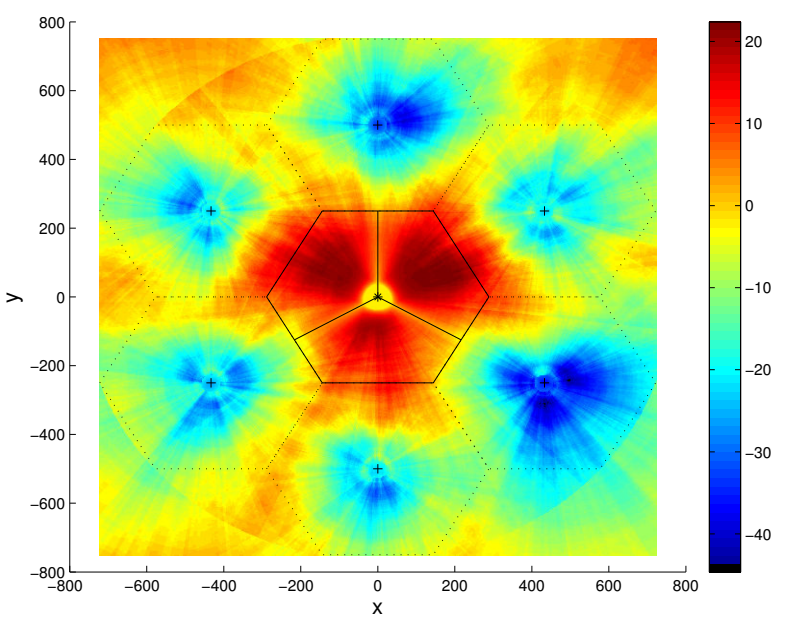

(b) LOS SINR with correlated shadowing.

\section{REFERENCES}

[1] J. Gozalvez, "Mobile traffic expected to grow more than 30-fold," IEEE Vehicular Technology Magazine, vol. 6, no. 3, pp. 9-15, 2011.

[2] A. Ghosh, R. Ratasuk, B. Mondal, N. Mangalvedhe, and T. Thomas, "LTE-Advanced: next-generation wireless broadband technology [invited paper]," IEEE Wireless Communications Magazine, vol. 17, no. 3, pp. $10-22,2010$.

[3] G. Hardin, "The tragedy of the commons," Science, vol. 162, pp. 1243 1248,1968

[4] ITU-R, "Report ITU-R M.2135-1; Guidelines for evaluation of radio interface technologies for IMT-Advanced," 2009.

[5] R. Schoenen, H. Yanikomeroglu, and B. Walke, "User-in-the-loop: Mobility aware users substantially boost spectral efficiency of cellular OFDMA systems," IEEE Communications Letters, vol. 15, no. 5, pp. 488-490, May 2011.

[6] R. Schoenen, G. Bulu, A. Mirtaheri, and H. Yanikomeroglu, "Green communications by demand shaping and User-in-the-Loop tariff-based control," in Proc. IEEE Online Green Communications Conference (GreenCom'11), Online, Sept. 2011.

[7] R. Schoenen, "User-in-the-loop project." [Online]. Available; http://www.userintheloop.org

[8] _ "On increasing the spectral efficiency more than $100 \%$ by user-inthe-control-loop," in Proc. Asia-Pacific Conference on Communications (APCC), Auckland, New Zealand, Oct. 2010.

[9] D. Bültmann, T. Andre, and R. Schoenen, "Analysis of 3GPP LTEAdvanced cell spectral efficiency," in Proc. IEEE International Symposium on Personal, Indoor and Mobile Radio Communications (PIMRC), Istanbul, Turkey, Sept. 2010.

[10] S. Szyszkowicz, F. Alaca, H. Yanikomeroglu, and J. Thompson, "Aggregate interference distribution from large wireless networks with correlated shadowing: An analytical-numerical-simulation approach," IEEE Transactions on Vehicular Technology, vol. 60, no. 6, 2011.

[11] F. Graziosi and F. Santucci, "A general correlation model for shadow fading in mobile radio systems," IEEE Communications Letters, vol. 6, no. 3, pp. 102-104, 2002.

[12] M. Gudmundson, "Correlation model for shadow fading in mobile radio systems," Electronics Letters, vol. 27, no. 23, pp. 2145-2146, 1991.

[13] K. Kumaran, S. Golowich, and S. Borst, "Correlated shadow-fading in wireless networks and its effect on call dropping," Wireless Networks, vol. 8, no. 1, pp. 61-71, 2002.

[14] I. Forkel, M. Schinnenburg, and M. Ang, "Generation of twodimensional correlated shadowing for mobile radio network simulation," in Proc. International Symposium on Wireless Personal Multimedia Communications (WPMC), Abano Terme, Italy, Sept. 2004.

[15] S. Szyszkowicz, F. Alaca, H. Yanikomeroglu, and J. Thompson, "Efficient simulation using shadowing fields of many wireless interferers with correlated shadowing," in Proc. IEEE Vehicular Technology Conference, Taipei, Taiwan, May 2010.

[16] R. Schoenen, G. Bulu, A. Mirtaheri, T. Beitelmal, and H. Yanikomeroglu, "Quantified user behavior in user-in-the-loop spatially and demand controlled cellular systems," in Proc. European Wireless Conference (EW), Poznan, Poland, April 2012. 